\title{
VOLATILIDADE DOS RETORNOS ECONÔMICOS ASSOCIADOS À INTEGRAÇÃO LAVOURA-PECUÁRIA NO ESTADO DO PARANÁ ${ }^{1}$
}

\author{
Joelsio José Lazzarotto ${ }^{2}$ \\ Maurinho Luiz dos Santos ${ }^{3}$ \\ João Eustáquio de Lima ${ }^{4}$ \\ Aníbal de Moraes ${ }^{5}$
}

Resumo: No setor agropecuário brasileiro, há grande diversidade e heterogeneidade de sistemas de produção, que tendem a ser cercados por amplo número de fatores de riscos. Embora não possam ser completamente eliminados, os riscos podem ser minimizados mediante adoção de algumas estratégias, como a diversificação com atividades agrícolas e pecuárias. Assim, partindo de resultados históricos de pesquisas para a agropecuária do estado do Paraná e utilizando, como medida principal, o valueat-risk (ou valor em risco), analisou-se a volatilidade dos retornos econômicos de sistemas com integração lavoura-bovinocultura de corte, diante de sistemas especializados na produção de grãos ou na bovinocultura de corte. As análises apontaram que, no curto prazo, a integração tende a gerar melhores resultados econômicos que os demais sistemas analisados.

Palavras-chave: sistemas de produção agropecuária, risco, value-at-risk.

\section{Introdução}

No setor agropecuário brasileiro, há grande diversidade e heterogeneidade de sistemas de produção, que tendem a ser cercados por amplo número

Recebido em: 06/04/09; Aceito em 13/08/09.

D.Sc. em Economia Aplicada. Pesquisador da EMBRAPA. E-mail: joelsio@cnpso.embrapa.br.

3 D.Sc. em Economia. Professor do Departamento de Economia Rural, da Universidade Federal de Viçosa. Email: mlsantos@ufv.br.

4 Ph.D. em Economia Rural. Professor do Departamento de Economia Rural, da Universidade Federal de Viçosa. E-mail: jelima@ufv.br.

5 D.Sc. em Zootecnia. Professor do Departamento de Fitotecnia e Fitossanitarismo, da Universidade Federal do Paraná. E-mail: anibalm@ufpr.br. 
de fatores de riscos operacionais e de mercado. Embora não possam ser completamente eliminados, esses riscos podem ser minimizados por meio de algumas estratégias, como a diversificação agropecuária.

Apesar de essa diversificação ter grande potencial para diminuir os riscos, destaca-se que, no Brasil, práticas de especialização na produção de certos produtos ainda são adotadas de forma generalizada. A condução de sistemas produtivos, baseados nessas práticas, pode resultar em problemas significativos tanto nos custos de produção como nos índices de produtividade (SANTOS et al., 1993).

A baixa diversificação de atividades pode, de certo modo, ser atribuída ao fato de que os produtores, para diversificarem seus sistemas produtivos, precisam de informações acerca da tecnologia e de quais atividades devem ser exploradas para capturar os reais benefícios em resultados econômicos (POPP; RUDSTROM, 2000). Nessa linha, considerando que a conjugação de atividades de produção animal e vegetal representa estratégia que pode trazer grandes benefícios técnicos e econômicos, está inserido este trabalho.

Operacionalmente, a integração lavoura-pecuária, que é uma estratégia de diversificação agropecuária, refere-se a sistemas dos quais participam atividades agrícolas e pecuárias, com um mínimo de interface entre si (MORAES et al., 2007). Esses sistemas podem ser muito vantajosos, pois, entre outras coisas, podem diversificar as fontes de receitas, diminuir riscos de frustrações de produção e, ou, de preços e reduzir impactos negativos ao meio ambiente.

No entanto, apesar de existirem, na literatura, diversos estudos que demonstram as vantagens técnicas em combinar explorações vegetais e animais, no Brasil, ainda é grande a carência de resultados que evidenciem os benefícios e riscos econômicos na condução de práticas de integração agropecuária. Adicionalmente, mediante leitura de alguns importantes trabalhos, como os de Kluthcouski et al. (2003) e Moraes et al. (2007), salienta-se que, no país, embora seja baixa a utilização de sistemas de 
integração lavoura-pecuária, existe enorme área potencial para exploração destes, durante todos os meses do ano.

Diante dessas inferências e com base na agropecuária paranaense ${ }^{6}$, buscou-se analisar, de forma comparativa e considerando situações de riscos operacionais e de mercado ${ }^{7}$, a volatilidade dos retornos econômicos de sistemas de produção com integração lavoura-bovinocultura de corte frente a sistemas caracterizados apenas pela exploração de grãos ou pela especialização na bovinocultura de corte.

\section{Fundamentação teórica}

Os pressupostos teóricos que nortearam a realização deste trabalho estão enquadrados em dois tópicos: a tomada de decisão sob condições de riscos e a teoria do portfólio.

\subsection{Tomada de decisão sob condições de riscos}

No processo de tomada de decisão dos investidores, os riscos exercem papeis fundamentais. Conceitualmente, o termo risco pode ser definido como uma medida do grau de incerteza acerca dos possíveis eventos futuros. Nesse caso, para quantificar o risco são utilizadas medidas estatísticas, em que se destacam as distribuições de probabilidade (REILLY; BROWN, 2003; GITMAN, 2004; TRAVERS, 2004).

É relevante enfatizar que, neste estudo, os riscos estão vinculados à noção dos retornos que podem ser obtidos mediante a realização de determinados investimentos. Genericamente, o termo retorno pode ser

\footnotetext{
6 Optou-se por trabalhar com o Paraná, por duas razões principais: 1) o Estado responde por expressiva parcela da produção agropecuária nacional; e 2) no Paraná, existem dados de pesquisas técnicas, de vários anos, acerca dos sistemas de integração lavoura-pecuária.

7 Neste estudo, a noção de risco operacional refere-se a possíveis problemas relativos às produtividades físicas das atividades agropecuárias. Por sua vez, riscos de mercado estão vinculados aos problemas decorrentes de flutuações que podem ocorrer, ao longo do tempo, nos preços pagos e recebidos pelos produtores rurais.
} 
visto como uma medida do total de ganhos (ou prejuízos), diante de dado nível de investimento (GITMAN, 2004).

Na prática, pelo fato de os investidores, em geral, não terem completa certeza sobre qual alternativa (ativo) de investimento proporcionará o maior retorno, eles tendem a tomar suas decisões com base no retorno esperado de cada alternativa. A estimativa do retorno esperado para determinado ativo corresponde à média ponderada dos possíveis retornos:

$$
E\left(R_{i}\right)=\sum_{j=1}^{n} p_{i j} R_{i j}
$$

em que $E\left(R_{i}\right)$ é o retorno esperado do ativo $i ; R_{i j}$ representa cada possível retorno desse ativo; e $p_{i j}$ corresponde à probabilidade de ocorrência de $R_{i j}$ (ELTON et al., 2003).

Segundo Markowitz (1952), representado pela variância, o risco mede a dispersão dos possíveis resultados de um experimento em torno de seu valor esperado (ou média). Assim, o risco de um ativo $i$, de acordo com Elton et al. (2003), pode ser representado da seguinte forma:

$$
\operatorname{var}\left(R_{i}\right)=\sum_{j=1}^{n}\left[p_{i j}\left(R_{i j}-E\left(R_{i}\right)\right)^{2}\right]
$$

em que $\operatorname{var}\left(R_{i}\right)$ é a variância do retorno do ativo $i ; E\left(R_{i}\right)$, retorno esperado desse ativo; $R_{i j}$, cada possível retorno do ativo; e $p_{i j}$, probabilidade de ocorrência de $R_{i j}$. 
Operacionalmente, é comum utilizar o desvio-padrão, que corresponde à raiz quadrada da variância, como medida do risco associado a um ativo. Portanto, com base no valor do desvio-padrão, pode-se avaliar o grau de incerteza vinculado a determinado investimento (REILLY; BROWN, 2003).

Essas considerações teóricas evidenciam a necessidade de os investidores, na tomada de decisões, avaliarem amplo número de fatores que podem afetar os resultados. Nessa perspectiva, com base na teoria do portfólio, a seguir, discorre-se sobre a otimização de carteiras de investimento, visando obter melhores relações em termos de retornos e riscos.

\subsection{A teoria do portfólio}

Segundo a teoria do portfólio, a diversificação de uma carteira de investimento pode constituir importante estratégia para reduzir os riscos. Isso porque os investidores podem combinar ativos que, ao longo do tempo, diminuam as flutuações no retorno esperado do portfólio, sem causar reduções significativas nesse retorno (HADAWAY, 1978).

Matematicamente, para uma carteira de investimento, o retorno esperado é dado pela média ponderada dos retornos esperados dos ativos individuais (ELTON et al., 2003):

$$
E\left(R_{p}\right)=\sum_{i=1}^{N} w_{i} E\left(R_{i}\right)
$$

em que $E\left(R_{p}\right)$ é o retorno esperado da carteira; $w_{i}$, participação do ativo $i$ na carteira, ou seja, a fração do portfólio que é investida no ativo 
$i$; e $E\left(R_{i}\right)$, retorno esperado desse ativo.

Com relação ao risco vinculado a uma carteira composta por $N$ ativos, ele pode ser avaliado a partir da variância dos retornos dessa carteira. Nesse caso, essa variância depende de componentes associados a duas medidas: as variâncias individuais dos retornos de cada ativo e as covariâncias entre os retornos dos ativos (MARKOWITZ, 1952):

$\operatorname{Var}\left(R_{p}\right)=\sum_{i=1}^{N} \operatorname{Var}\left(R_{i}\right) w_{i}^{2}+\sum_{i=1}^{N} \sum_{s=1}^{N} w_{i} w_{s} \operatorname{Cov}\left(R_{i}, R_{s}\right)$, sendo $i \neq s$,

em que $\operatorname{Var}\left(R_{p}\right)$ é a variância dos retornos da carteira; $\operatorname{Var}\left(R_{i}\right)$, variância dos retornos de cada ativo; $w_{i}$, participação de cada ativo no portfólio, sendo $\sum_{i=1}^{N} w_{i}=1$ e $w_{i} \geq 0$; e $\operatorname{Cov}\left(R_{i}, R_{s}\right)$, covariância entre os retornos de dois ativos.

Os componentes de covariâncias, destacados em (4), possibilitam avaliar, por meio do sinal e da magnitude, se os retornos de dois ativos apresentam, ao longo do tempo, comportamentos distintos (ou não). Nesse sentido, para que a diversificação de ativos possa resultar em maior efeito na minimização dos riscos, Markowitz (1952) enfatizou que os investidores deveriam investir em ativos que apresentam, entre si, altas covariâncias negativas.

\section{Metodologia}

Esta seção está estruturada em três partes principais. Na primeira, são feitas considerações acerca do objeto de estudo. Na parte dois, discorrese sobre a análise de volatilidade. A terceira parte trata das fontes de dados e dos recursos computacionais. 
Joelsio José Lazzarotto, Maurinho Luiz dos Santos,

João Eustáquio de Lima \& Aníbal de Moraes

\subsection{Considerações principais referentes ao objeto de estudo}

Para consecução do estudo, foram definidos três sistemas: 1) Produção vegetal, que corresponde ao sistema que ocorre quando, em determinada propriedade rural, são exploradas apenas atividades de produção de grãos (soja e milho no verão e trigo no inverno); 2) Bovinocultura de corte, que é o sistema que ocorre quando o estabelecimento agropecuário é especializado na produção de carne bovina, mediante a compra de animais (bezerros) para desenvolver as fases de recria e engorda, em período inferior a um ano; e 3) Integração lavoura-bovinocultura de corte, que constitui o sistema no qual, em distintos períodos do ano, existem produções sucessivas de grãos (soja e milho no verão e trigo no inverno) e carne bovina (verão e inverno), ou seja, ao longo de todo ano, há partes da área exploradas com atividades agrícolas e outras, com a pecuária, que também é caracterizada pela compra de bezerros para, em período inferior a um ano, desenvolver as fases de recria e engorda.

Com relação a esses sistemas, é relevante enfatizar que eles constituem modelos teóricos bem ajustados à realidade da microrregião de Guarapuava - $\mathrm{PR}^{8}$, pois foram estruturados mediante consultas a pesquisadores do Departamento de Fitotecnia e Fitossanitarismo da Universidade Federal do Paraná e da Cooperativa Agrária Agroindustrial, de Guarapuava - PR 9 . Essas instituições, há mais de uma década, vêm desenvolvendo uma série de pesquisas, principalmente de natureza técnica (o foco principal é a avaliação da estrutura técnica de produção), visando à identificação de diferentes alternativas de sistemas de produção que sejam adequados à realidade agropecuária do Paraná.

8 Essa microrregião, que tem na agropecuária a base principal para o seu desenvolvimento, é muito representativa da adoção de sistemas de integração lavoura-pecuária no Estado paranaense.

9 Neste estudo, presume-se que, tecnicamente, seja viável explorar cada um dos sistemas de produção definidos, ou seja, na prática, a escolha de qual sistema será explorado depende apenas das preferências do produtor rural. 


\subsection{Análise de volatilidade}

Após definidos os sistemas, realizou-se a análise de volatilidade, que consistiu em avaliar, no curto prazo, a variabilidade dos retornos econômicos das distintas alternativas de sistemas. Para medir a volatilidade, empregou-se a técnica do value-at-risk (VaR), que é considerada, por muitos, como o último passo na evolução das ferramentas de administração de risco (MANFREDO; LEUTHOLD, 1999; JORION, 2003; TRAVERS, 2004).

Em termos operacionais, considerando determinado nível de confiança e período de tempo preestabelecidos, o VaR, sob condições normais de mercado, mede a máxima perda monetária esperada, em decorrência de movimentos adversos nos preços que afetam os ativos de um portfólio (MANFREDO; LEUTHOLD, 1999; JORION, 2003).

É importante ressaltar que, embora o VaR tenha sido desenvolvido para utilização em instituições financeiras, atualmente, essa técnica vem sendo muito empregada em outras instituições (JORION, 2003). Nessa linha, Manfredo e Leuthold (1998) ressaltaram a existência de várias aplicações potenciais do uso do VaR na administração de riscos agropecuários.

Neste estudo, para chegar às estimativas do VaR, foram seguidos três passos principais: 1) Cálculo dos retornos econômicos das atividades agropecuárias comerciais, que compõem os três sistemas de produção agropecuária; 2) Estimação das matrizes de variâncias e covariâncias dos retornos; e 3) Finalmente, o cálculo do VaR.

Para obter os retornos, primeiramente foram calculadas as séries de receita total (RT), custo total (CT) e lucro total (LT). Para isso, utilizaramse preços históricos mensais recebidos e pagos pelos produtores, no período de janeiro de 1995 a agosto de 2007, totalizando 152 observações. Sobre essas séries, é importante salientar que, para fins de ampliação de dados amostrais, considerou-se que cada observação representava um suposto ano agrícola. Essa pressuposição é justificada por três razões: 1) 
A maior parte dos produtores, em cada ano agrícola, realizava apenas uma operação de venda relativa a cada um dos produtos agropecuários produzidos; 2) Em cada ano agrícola e para cada insumo, a maioria dos agricultores efetuava somente uma operação de compra; e 3) Dada a necessidade de conseguir amostras maiores, possibilitando, portanto, efetuar melhores estimativas acerca do comportamento e da volatilidade dos retornos econômicos ao longo do tempo.

Na elaboração das referidas séries foram também utilizadas informações técnicas dos sistemas, que se referem às produtividades e aos componentes tecnológicos (fatores de produção), empregados no ciclo produtivo. A respeito das produtividades, foram consideradas duas situações. Na primeira, para todas as 152 observações, utilizaram-se as produtividades históricas médias das atividades agropecuárias; na segunda, com base na distribuição de probabilidade triangular ${ }^{10}$, para cada ano agrícola, foram gerados, por meio de simulação, 100 diferentes valores de produtividades. Assim, enquanto o uso de produtividades constantes possibilitou desenvolver análises que envolvem apenas os impactos de fatores de mercado sobre os desempenhos econômicos dos sistemas, o uso de produtividades variáveis permitiu avaliar os efeitos conjuntos decorrentes de mudanças em fatores operacionais e de mercado sobre os referidos desempenhos.

Matematicamente, as séries obtidas de LT podem ser representadas da seguinte forma:

$$
L T_{j t}=R T_{j t}-C T_{j t}
$$

em que $L T_{j t}$ é o lucro total da atividade $j$, no período $t ; R T_{j t}$, receita total dessa atividade, que é resultante da multiplicação do preço de venda

\footnotetext{
${ }^{10}$ Foi utilizada a distribuição triangular devido ao fato de que, segundo Moura (2004), quando existe falta de informações que permitam um ajustamento mais criterioso das distribuições, ela, em geral, é aceita como satisfatória. A principal vantagem é sua facilidade de uso, pois, para defini-la, são necessários apenas três valores: mínimo, máximo e mais provável da variável.
} 
pela produtividade do produto no período $t$; e $C T_{j t}$, custo total da atividade $j$, formado pelos custos fixos e variáveis, no período $t$.

Os custos fixos são representados pelo somatório dos valores anuais, associados, principalmente, com mão-de-obra permanente, depreciação, seguro e manutenção dos bens de capital. Por outro lado, os custos variáveis correspondem ao somatório dos gastos com insumos consumidos no processo produtivo, operações agrícolas e outros itens, como mãode-obra temporária, assistência técnica, recepção do produto e contribuição especial da Seguridade Social Rural (CESSR). Utilizando uma TMA real de 12,0\% a.a., aos custos fixos e variáveis foram também adicionados, respectivamente, os custos de oportunidade dos capitais imobilizado e mobilizado (capital consumido no processo produtivo).

Com as séries de CT e LT, foram obtidos os retornos esperados, expressos pela relação LT/CT, para os três sistemas. Esses retornos são representados pela seguinte equação:

$$
E\left(R_{S t}\right)=\sum_{j=1}^{N} w_{j} R_{j t}, \text { sendo } R_{j t}=\frac{L T_{j t}}{C T_{j t}}, \sum_{j=1}^{N} w_{j}=1 \text { e } w_{j} \geq 0
$$

em que $E\left(R_{s t}\right)$ é o retorno esperado, no período $t$, do sistema de produção composto por $N$ atividades agropecuárias comerciais; $w_{j}$, participação média do CT de cada atividade no custo total do sistema; e $R_{j t}$, retorno da atividade $j$, no período $t$.

Com base nas séries de retornos obtidas com produtividades constantes, foram estimadas as matrizes Var-Cov dos retornos econômicos de cada sistema:

$$
\operatorname{Var}-\operatorname{Cov}=\sigma_{p}^{2}=\sum_{j=1}^{N} \sum_{i=1}^{N} w_{j} w_{i} \sigma_{j i}
$$


em que $\sigma_{p}^{2}$ é a variância dos retornos de cada sistema; $w_{j}$, participação média do CT de cada atividade no CT do sistema; e $\sigma_{j i}$, covariância entre os retornos das atividades $j e i$.

A partir dos retornos e das estimativas das Var-Cov, foram gerados os resultados do VaR dos sistemas, por meio dos métodos de simulação histórica com produtividades constantes (HPC) e variáveis (HPV) e delta normal (DN). Nos métodos de simulação, as séries efetivamente observadas dos retornos foram ordenadas, de forma crescente. Após esse ordenamento, obteve-se, para cada série, o retorno correspondente ao percentil de 5\%; e o valor obtido representa o pior retorno esperado para o nível de confiança de $95 \%$. Com relação ao método delta normal, para calcular o VaR a partir das variâncias e covariâncias, admitiu-se que os retornos das atividades que compunham os sistemas fossem normalmente distribuídos.

É importante destacar que a utilização dos métodos de simulação e delta normal é plenamente justificada. Isso porque, dadas as distribuições de probabilidade específicas dos retornos estudados, pode-se, mediante o emprego do primeiro e segundo métodos, minimizar a ocorrência de possíveis problemas de estimativas do VaR, associadas com retornos que sigam, respectivamente, distribuições não normal e normal (KONDAPANENI, 2005).

As expressões (8) e (9) representam as equações efetivamente usadas para calcular o VaR, que, neste trabalho, representa o pior lucro total que o sistema de produção pode apresentar, em um ano agrícola, a um nível de confiança de $95 \%$.

$$
\begin{aligned}
& \operatorname{VaR}_{\text {Histórico }}=C T_{\text {Médio }} \times R^{*}, \\
& V a R_{D N}=C T_{\text {Médio }} \times(\mu-1,65 \sigma),
\end{aligned}
$$


em que $C T_{\text {Médio }}$ é o CT médio de cada sistema; $R^{*}$, retorno crítico, com percentil 5\%, do sistema; $\mu$, retorno médio da série de retornos de cada sistema; $\sigma$, desvio-padrão relativo à série de retornos de cada sistema; e 1,65 indica, para uma distribuição normal padronizada, o número de desvios-padrão para o nível de confiança unicaudal de $95 \%$.

Para avaliar a acurácia das estimativas do VaR, utilizaram-se dois testes (Kupiec e $Z^{11}$ ), que visavam analisar o número de violações, ou seja, avaliar o número de períodos em que o LT foi menor que a estimativa do VaR. Neste trabalho, eram esperadas $5 \%$ de violações.

Após obter as estimativas do VaR, com base em procedimentos similares aos empregados por Costa (2003), para cada sistema calculou-se o Índice Sharpe Adaptado (ISA), que é resultante da divisão do VaR pelo retorno esperado. O ISA permitiu analisar, para cada configuração possível de sistema, a relação entre risco e retorno esperados.

\subsection{Fontes de dados e recursos computacionais}

As informações técnicas para estruturação dos modelos de sistemas estudados foram provenientes de resultados de pesquisas gerados pelo Departamento de Fitotecnia e Fitossanitarismo da Universidade Federal do Paraná e pela Cooperativa Agrária Agroindustrial de Guarapuava - PR.

Quanto aos dados históricos relativos aos preços recebidos e pagos, eles foram obtidos no Departamento de Economia Rural da Secretaria da Agricultura e do Abastecimento do Estado do Paraná. Esses preços foram deflacionadas com o IGP-DI, para março de 2008.

Em relação aos recursos computacionais, além da planilha eletrônica Microsoft Excel, utilizaram-se os softwares @Risk e BestFit, desenvolvidos pela Palisade Corporation.

11 Para maiores detalhes a respeito dos testes Kupiec e Z, pode-se consultar Goorbergh e Vlaar (1999) e Manfredo e Leuthold (1999). 


\section{Resultados e discussão}

Esta seção está organizada em duas partes. Na primeira, efetua-se uma breve caracterização dos três sistemas: produção vegetal (PV), integração lavoura-pecuária (ILP) e bovinocultura de corte (BC). Na segunda, realiza-se a análise de volatilidade.

\subsection{Caracterização dos sistemas de produção}

Considerando que a disponibilidade de terra é condição imprescindível para a exploração agropecuária, foi fixada, para cada sistema, uma área de 300 hectares, que é ocupada, ao longo do ano, com atividades comerciais e atividades-meio ${ }^{12}$. No Quadro 1 , ao serem comparados os sistemas de PV e ILP, constata-se que as áreas comerciais das atividades agrícolas são menores no sistema de integração, pois é necessário estabelecer áreas de pastagem permanente e de cultivo de silagem, que são destinadas à bovinocultura de corte.

Na composição dos sistemas, verifica-se ainda que, durante o inverno, enquanto na ILP se utilizava toda a área com finalidade comercial, no sistema de PV apenas 33,3\% da área era explorada para esse fim. Esse dado é plenamente justificado, pois, na região de Guarapuava, os produtores que adotavam o sistema de PV e que apenas cultivavam, de forma comercial, trigo no inverno, tendiam a explorar a triticultura em somente parte da área. Esse uso parcial deve-se a problemas de rentabilidade e de riscos climáticos que, comumente, cercam essa cultura.

Ao analisar o sistema de BC, percebe-se que ele é especializado em uma atividade comercial. Adicionalmente, a comparação entre os sistemas de BC e PV mostra que eles apresentam características estruturais e funcionais muito distintas. Enquanto no sistema de BC toda a área

\footnotetext{
${ }_{12}$ Neste estudo, as explorações de carne bovina, milho, soja e trigo são definidas como atividades comerciais, pois têm como finalidades principais obter produtos comercializáveis. Entretanto, as pastagens permanente e cultivada, o milho para silagem e o confinamento representam as atividades-meio, que visam viabilizar, tecnicamente, as produções relacionadas com as atividades comerciais dos três sistemas.
} 
agropecuária é direcionada para a pecuária, no sistema de PV essa área é exclusiva para as atividades agrícolas. No entanto, ao comparar os sistemas de BC e ILP, também são percebidas importantes diferenças; enquanto o primeiro é o mais especializado, o de ILP é o mais diversificado dos três sistemas.

Quadro 1 - Atividades comerciais, atividades-meio e indicadores técnicos nos três sistemas

\begin{tabular}{|c|c|c|c|c|c|}
\hline & Item & Período/Medida & PV & ILP & BC \\
\hline \multirow{4}{*}{ 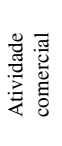 } & Soja (ha) & Verão & 200,0 & 160,0 & 0,0 \\
\hline & Milho (ha) & Verão & 100,0 & 80,0 & 0,0 \\
\hline & Trigo (ha) & Inverno & 100,0 & 80,0 & 0,0 \\
\hline & Bovinocultura de corte (cab) & $\mathrm{cab}$ & 0 & 495 & 1.350 \\
\hline \multirow{4}{*}{ 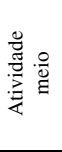 } & Pastagem permanente de tifton (ha) & Verão & 0,0 & 52,1 & 278,5 \\
\hline & Pastagem cultivada de aveia/azevém (ha) & Inverno & 200,0 & 220,0 & 300,0 \\
\hline & Milho para silagem (ha) & Verão & 0,0 & 7,9 & 21,5 \\
\hline & Confinamento (cab) & $\mathrm{cab}$ & 0 & 495 & 1.350 \\
\hline \multirow{15}{*}{ 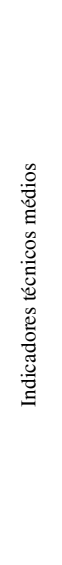 } & Produtividade média de soja & $\mathrm{kg} / \mathrm{ha}$ & 3.000 & 3.000 & -- \\
\hline & Produtividade média de milho & $\mathrm{kg} / \mathrm{ha}$ & 8.500 & 8.500 & -- \\
\hline & Produtividade média de trigo & $\mathrm{kg} / \mathrm{ha}$ & 2.500 & 2.500 & -- \\
\hline & Peso de aquisição dos bezerros & $\mathrm{kg}$ de peso vivo & -- & 180 & 180 \\
\hline & Peso de venda dos animais para o abate & $\mathrm{kg}$ de peso vivo & -- & 450 & 450 \\
\hline & Fase de recria (pastagens) & dia & -- & 270 & 270 \\
\hline & Fase de engorda (confinamento) & dia & -- & 45 & 45 \\
\hline & Tempo total (recria e engorda) & dia & -- & 315 & 315 \\
\hline & Ganho de peso total/animal & $\mathrm{kg}$ de peso vivo & -- & 270 & 270 \\
\hline & Ganho médio de peso vivo & $\mathrm{kg} / \mathrm{animal} / \mathrm{dia}$ & -- & 0,86 & 0,86 \\
\hline & Lotação na pastagem permanente (verão) & animais/ha & -- & 9,50 & 4,85 \\
\hline & Lotação na pastagem cultivada (inverno) & animais/ha & -- & 2,25 & 4,50 \\
\hline & Total de animais & $\mathrm{cab}$ & -- & 495 & 1.350 \\
\hline & Rendimento de carcaça & $\%$ & -- & 53,0 & 53,0 \\
\hline & Taxa de mortalidade de animais & $\%$ & -- & 1,0 & 1,0 \\
\hline
\end{tabular}

Fonte: Pesquisa de campo.

Notas: 1) Neste trabalho, os períodos de verão e inverno representam, respectivamente, os períodos de novembro a abril e de maio a outubro; e 2) As lotações de 9,5 e 4,5 animais/ha, respectivamente, nas pastagens permanente e cultivada, estão próximas aos limites superiores, a partir dos quais as pastagens estão mais sujeitas a apresentar problemas de desenvolvimento, associados, por exemplo, a compactação do solo em períodos mais chuvosos. 
É relevante assinalar que, nos sistemas de ILP e BC, a bovinocultura de corte é explorada de maneira tecnológica muito similar, isto é, nos dois sistemas, as fases de recria e engorda ${ }^{13}$ dos animais são efetuadas por meio da utilização de pastagens e confinamento. A maior diferença na condução da atividade de pecuária, nos dois sistemas, reside, principalmente, no tamanho da exploração; à medida que no sistema de ILP são adquiridos, anualmente, cerca de 495 bezerros, no sistema de $\mathrm{BC}$ esse número é da ordem de 1.350. Com esses números, busca-se manter lotações que não resultem em problemas técnicos, como compactação do solo e baixa oferta de alimentos para animais.

Em relação às atividades-meio, é pertinente destacar alguns pontos:

- No verão, a pastagem permanente, que tem vida útil de cerca de 15 anos, visa, basicamente, fornecer alimentos para os animais;

- No inverno, a pastagem cultivada com aveia e azevém, que é implantada sobre áreas de grãos e de pastagem permanente, possui diferentes objetivos; no sistema de $P V$, visa produzir biomassa para a cobertura do solo e o uso da técnica de plantio direto de grãos; no sistema de ILP, além dos objetivos destacados para o sistema de PV, busca produzir alimentos para os bovinos; e no sistema de $B C$, atender à demanda alimentar dos animais;

- A produção de milho para silagem, que está presente nos sistemas que possuem bovinos de corte, tem o objetivo de fornecer alimentos para os animais na fase de engorda; e

- A fase de engorda é realizada em confinamento baseado em silagem e concentrado. O confinamento, que visa garantir com que os animais atinjam o peso de abate planejado e com adequado acabamento de carcaça, é realizado durante cerca de 45 dias.

13 Enquanto a fase de recria compreende o período desde a aquisição dos animais (aproximadamente $180 \mathrm{~kg}$ de peso vivo) até o início da fase de engorda (peso vivo em torno de $400 \mathrm{~kg}$ ), esta termina quando os animais atingem cerca de $450 \mathrm{~kg}$ de peso vivo. 
Em termos tecnológicos, pode-se inferir que as atividades desenvolvidas nos três sistemas são altamente tecnificadas. De acordo com o Quadro 1 , e utilizando como referência os valores das produtividades agrícolas médias, estimadas pela Conab (2008) para o Paraná ${ }^{14}$, verifica-se que os sistemas de PV e ILP tendem a apresentar elevadas produtividades.

Com base no Quadro 1, observa-se, ainda, que os sistemas de PV e ILP apresentam as mesmas produtividades agrícolas, visto que, embora apresentem algumas diferenças importantes na composição e na dinâmica de funcionamento, a tecnologia empregada na exploração das atividades agrícolas é similar nos dois sistemas.

Quanto à exploração da bovinocultura de corte, no Quadro 1 também estão os principais indicadores técnicos associados com esta atividade, presente nos sistemas de ILP e BC. A tecnologia empregada na produção de bovinos de corte é também muito similar nos dois sistemas supracitados. As diferenças técnicas fundamentais na condução da exploração estão associadas às taxas de lotação das pastagens permanente e cultivada.

De maneira geral, pode-se assinalar que o excelente desempenho das atividades agropecuárias, exploradas nos três sistemas, deve-se a três fatores principais: 1) Na região de Guarapuava (PR), existem condições agroecológicas (terra e clima) muito favoráveis ao desenvolvimento da agropecuária de alto rendimento técnico; 2) Os produtores que desenvolvem esses sistemas, geralmente, são propensos a empregar altos níveis tecnológicos; e 3) Há, na região estudada, adequado suporte de assistência técnica.

${ }^{14}$ Na última década, as produtividades médias de soja, milho e trigo no Paraná foram, respectivamente, de 2.713, 4.949 e $1.767 \mathrm{~kg} / \mathrm{ha}(\mathrm{Conab}, 2008)$. 


\subsection{A volatilidade dos retornos econômicos dos sistemas de produção}

No Quadro 2, são apresentados os resultados de valores esperados para os três sistemas de produção. Com base nesses resultados, podem-se fazer três comentários acerca dos desempenhos econômicos médios dos sistemas: 1) Tanto com produtividades constantes como variáveis, os retornos para os sistemas de PV e ILP são muito próximos; 2) O sistema de BC é o que apresenta o menor retorno esperado, em ambas as situações; e 3) Quando as produtividades variam, nos três sistemas, observam-se apenas pequenas oscilações em relação aos resultados obtidos com produtividades constantes.

Quadro 2 - Valores anuais esperados para os sistemas de PV, ILP e BC

\begin{tabular}{|c|c|c|c|c|c|c|c|c|}
\hline \multirow{2}{*}{ Sistema } & \multicolumn{4}{|c|}{$\begin{array}{l}\text { Valores esperados com produtividades } \\
\text { constantes }\end{array}$} & \multicolumn{4}{|c|}{ Valores esperados com produtividades variáveis } \\
\hline & $\begin{array}{c}\text { RT } \\
(\mathbf{R} \$)\end{array}$ & $\begin{array}{l}\text { CT } \\
\text { (R\$) }\end{array}$ & $\begin{array}{c}\text { LT } \\
(\mathbf{R} \$)\end{array}$ & $\begin{array}{c}\text { LT/CT } \\
(\%)\end{array}$ & $\begin{array}{c}\text { RT } \\
(\mathbf{R} \$)\end{array}$ & $\begin{array}{c}\text { CT } \\
\text { (R\$) }\end{array}$ & $\begin{array}{c}\text { LT } \\
\text { (R\$) }\end{array}$ & LT/CT (\%) \\
\hline $\mathrm{PV}$ & 786.339 & 711.747 & 74.592 & 10,5 & 780.031 & 711.436 & 68.595 & 9,6 \\
\hline ILP & 1.181 .381 & 1.070 .060 & 111.322 & 10,4 & 1.176 .890 & 1.070 .472 & 106.418 & 9,9 \\
\hline $\mathrm{BC}$ & 1.506 .035 & 1.445 .856 & 60.178 & 4,2 & 1.507 .435 & 1.447 .660 & 59.776 & 4,1 \\
\hline
\end{tabular}

Fonte: Resultados da pesquisa.

No que concerne às análises realizadas a partir do Quadro 2, deve-se destacar que elas são muito limitadas, especialmente por desconsiderar as possíveis variações que, ao longo do tempo, podem ser observadas nos resultados dos distintos sistemas. Diante disso, a seguir, são desenvolvidas análises em que são levadas em conta as variabilidades de desempenho.

Na Figura 1, têm-se as séries de retornos econômicos, com produtividades constantes, dos três sistemas. Visualmente, percebe-se que os retornos apresentam grandes variações ao longo do tempo. $\mathrm{O}$ fato de as variações mais acentuadas serem evidenciadas no sistema de PV permite inferir que as atividades agrícolas (soja, milho e trigo), quando em situações de mercado favoráveis (maior relação preços recebidos/preços pagos), propiciam altos retornos, superando os dos sistemas em que a atividade 
de pecuária está presente. No entanto, em situações de mercado desfavoráveis, as atividades agrícolas tendem a ser mais afetadas negativamente, provocando, assim, acentuadas quedas nos retornos do sistema. Essas constatações, de certa maneira, condizem com as de Canziani e Guimarães (2007), pois, ao estudarem a viabilidade econômica da pecuária de corte no sistema de ILP no Paraná, por meio da utilização de dados mensais que compreendem o período de 1996 a 2006, observaram que, quando os preços pagos pelos produtos agropecuários (milho, trigo e boi gordo) estão baixos, a pecuária aparece como alternativa mais rentável, especialmente para a safra de inverno. No entanto, nas situações em que todos os preços desses produtos estão altos, as atividades agrícolas, em geral, resultam em maiores retornos em relação à pecuária.

O sistema de $\mathrm{BC}$, apesar de apresentar o menor retorno esperado, é aquele que, visualmente, possui a série de retornos com menos clusters de volatilidade, ou seja, não se visualizam claramente, em comparação com as séries de retornos dos outros dois sistemas, períodos com pequenas oscilações alternados com períodos de grandes oscilações nos retornos. Com isso, ao incluir a atividade de pecuária no sistema que antes era especializado na produção vegetal, reduz-se o tamanho das variações na série de retornos. Isso porque, conforme se verifica na Figura 1, a série de retornos do sistema de ILP tende a se situar em uma posição intermediária entre as séries de retornos dos outros dois sistemas. 


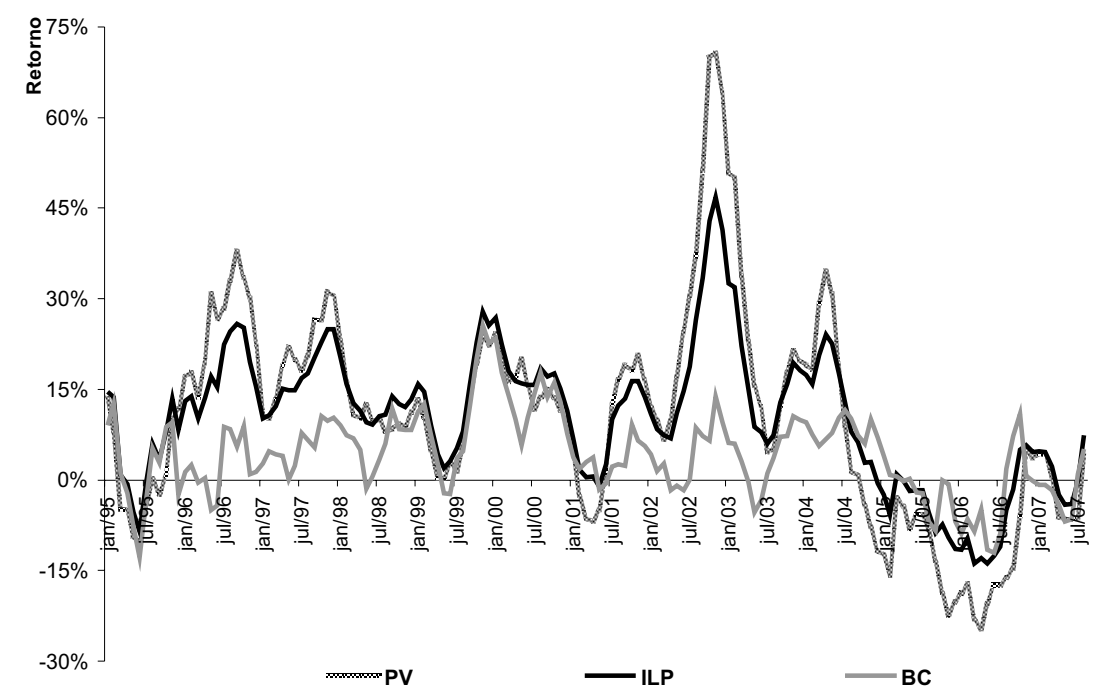

Fonte: Resultados da pesquisa.

Figura 1 - Retornos (LT/CT), com produtividades constantes, dos três sistemas.

Com relação às séries de retornos, foram calculadas também as distribuições de frequências relativas (FR). Ao analisar a Figura 2, percebe-se claramente que, enquanto o sistema de $\mathrm{BC}$ possui os retornos distribuídos mais próximos do valor esperado, no sistema de PV há maior amplitude de variação em relação a esse valor. Contudo, embora o sistema de BC apresente, visualmente, retornos menos dispersos em relação aos dos outros dois sistemas, é possível perceber que, na ILP, os retornos se distribuem de forma mais favorável, pois, se por um lado o retorno mínimo calculado está muito próximo daquele associado com o do sistema de BC (-13,9\% e $12,8 \%$, respectivamente), por outro, o valor máximo é bastante superior ao observado nesse sistema (46,8\% e $25,6 \%$, respectivamente).

A partir das representações das frequências relativas, evidencia-se, ainda, que o sistema de ILP, em comparação com o de PV, apresenta menor variabilidade nos seus retornos. Isso porque, para retornos médios muito semelhantes $(10,4 \%$ e $10,5 \%)$, os dois conjuntos de dados são claramente diferentes, sendo muito mais dispersos no sistema de PV. 


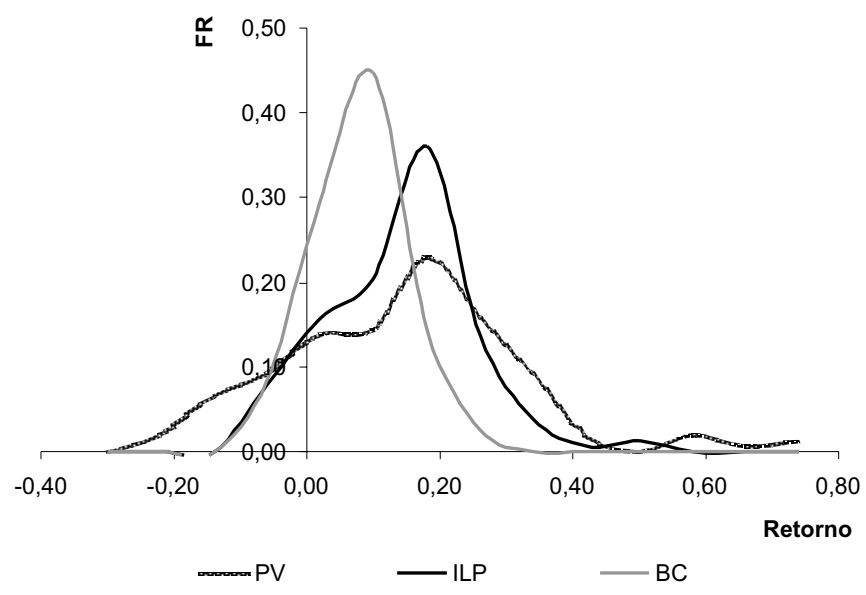

Fonte: Resultados da pesquisa.

Figura 2 - Representações das frequências relativas das séries de retornos (LT/CT), com produtividades constantes.

Em síntese, as Figuras 1 e 2 sinalizam que, em termos econômicos, a combinação de atividades agrícolas e de pecuária pode trazer vantagens interessantes, especialmente por propiciar, no curto prazo, adequados retornos e reduzir a instabilidade na renda do produtor rural. No entanto, para dar suporte às discussões já efetuadas, e tendo em vista que os resultados econômicos dependem, em muito, das produtividades, é necessário efetuar análises em que se admitem desempenhos técnicos variados e, também, considerem as possíveis perdas monetárias. Nessa perspectiva, utilizando como medida o value-at-risk, foi elaborado o Quadro 3, no qual, ao admitir tanto a ocorrência de produtividades constantes como variáveis, são apresentadas estimativas probabilísticas dos piores lucros esperados ( $\mathrm{VaR})$, em termos monetários e percentuais, que podem ser observados em um ano agrícola.

Com base nas estimativas apresentadas no Quadro 3, é pertinente ressaltar cinco resultados: 1) O sistema de PV está sujeito a apresentar os maiores prejuízos, pois, na média das três estimativas, o VaR foi de - 
$\mathrm{R} \$ 129.111$ (retorno de $-18,1 \%$ ); 2) Enquanto no sistema de ILP foi registrado o menor prejuízo monetário (o VaR médio foi de $-\mathrm{R} \$ 100.769$ ), no de $\mathrm{BC}$ estimou-se o menor prejuízo médio relativo $(-7,6 \%) ; 3)$ Os três métodos usados para realizar as estimativas (HPC, DN e HPV), além de apresentarem acurácia significativa, geraram resultados que conduzem às mesmas conclusões; 4) Ao serem comparados os valores do VaR, obtidos com produtividades constantes e varáveis, percebem-se apenas pequenas variações, e os desempenhos econômicos estão ligeiramente piores na situação de produtividades variáveis; e 5) O sistema de ILP tende a propiciar, ao longo do tempo, melhor relação risco/retorno, pois, ao analisar as médias do ISA de cada sistema, pode-se dizer que, na ILP, para cada unidade de retorno, há apenas 0,920 unidade de risco associada.

Quadro 3 - Estimativas do value-at-risk (VaR) e da relação risco/ retorno, medida pelo ISA, para cada sistema de produção

\begin{tabular}{|cccccc|}
\hline Sistema & Método & VaR $(\mathbf{R} \$)$ & VaR $(\%)$ & ISA & ISA médio \\
\hline \multirow{4}{*}{ PV } & HPC & $-123.710,9$ & $-17,4$ & 1,658 & \\
& DN & $-130.594,1$ & $-18,3$ & 1,751 & 1,783 \\
& HPV & $-133.028,1$ & $-18,7$ & 1,939 & \\
\hline \multirow{3}{*}{ ILP } & HPC & $-102.276,4$ & $-9,6$ & 0,919 & \\
& DN & $-89.407,8$ & $-8,4$ & 0,803 & 0,920 \\
& HPV & $-110.621,4$ & $-10,3$ & 1,039 & \\
\hline \multirow{3}{*}{ BC } & HPC & $-105.244,7$ & $-7,3$ & 1,749 & \multirow{2}{*}{1,837} \\
& DN & $-104.837,4$ & $-7,3$ & 1,742 & \\
& HPV & $-120.681,3$ & $-8,3$ & 2,019 & \\
\hline
\end{tabular}

Fonte: Resultados da pesquisa. 


\section{Conclusões}

Os sistemas de produção agropecuária estudados, embora apresentem muitos componentes tecnológicos em comum, possuem diferenças bastante expressivas entre si. Essas diferenças devem-se, sobretudo, à composição das atividades. Enquanto o sistema de BC é especializado apenas na atividade de pecuária e o sistema de PV só contempla atividades agrícolas, o de ILP é o mais diversificado, pois possui todas as atividades agropecuárias presentes nos outros dois sistemas. Adicionalmente, destaca-se que a ILP tende a ser o sistema mais complexo, pois exige, por parte do produtor rural, maior conjunto de conhecimentos técnicos e mercadológicos, associados com atividades agrícolas e pecuárias.

Os resultados da análise de volatilidade mostraram que o desempenho econômico dos três sistemas de produção pode, entre intervalos de um ano agrícola, variar significativamente. Entretanto, ao comparar os resultados observados em cada sistema, é possível afirmar que, no curto prazo, a ILP propicia melhores resultados. Mesmo em situações desfavoráveis, vinculadas com aspectos técnicos e, ou, mercadológicos, esse sistema, com base nas medidas de retorno esperado e do value-atrisk, apresenta a relação mais favorável entre retornos e riscos. Por sua vez, o sistema de PV é o que apresenta retornos econômicos mais voláteis, quando se admite um horizonte temporal de um ano agrícola.

Portanto, em termos econômicos, é possível inferir que a combinação de atividades agrícolas e de pecuária pode trazer vantagens interessantes, como redução na relação risco/retorno esperados. 


\section{Referências}

CANZIANI, J. R.; GUIMARÃES, V. D. A. Análise da viabilidade econômica da pecuária de corte no "sistema de integração lavourapecuária" em substituição às culturas de trigo e milho safrinha no Estado do Paraná. In.: SIMPÓSIO INTERNACIONAL EM INTEGRAÇÃO LAVOURA-PECUÁRIA, 2007. Palestras..., Curitiba: UFPR/UFRGS/ OHIO STATE, 2007. 16p. 1 CD-ROM.

CONAB - COMPANHIA NACIONAL DE ABASTECIMENTO. Séries históricas de produtividade de grãos: safra 1976/77 a 2006/ 07. Disponível em: <http://www.conab.gov.br/conabweb/ index.php?PAG=131>. Acesso em: 13 de julho de 2008.

COSTA, T. M. T. Viabilidade da utilização de derivativos agropecuários em carteiras de investimentos de Fundos de Pensão no Brasil. 2003. 102f. Dissertação (Mestrado em Economia Aplicada) - Universidade Federal de Viçosa, Viçosa, 2003.

ELTON, E. J.; GRUBER, M. J.; BROWN, S. J.; GOETZMANN, W. N. Modern portfolio theory and investment analysis. 6.ed. New York: John Wiley \& Sons, 2003. 705p.

GITMAN, L. J. Princípios de administração financeira. 10.ed. São Paulo: Pearson Addison Wesley, 2004. 745p.

GOORBERGH, R. van den; VLAAR, P. Value-at-Risk analysis of stock returns: historical simulation, variance techniques or tail index estimation? Amsterdam: Netherlands Central Bank, 1999. 37p. (DNB Staff Reports, n.40). Disponível em: <http://www.smartquant.com/ references/VaR/var46.pdf>. Acesso em: 22 de junho de 2007.

HADAWAY, S. C. Diversification possibilities in agricultural land investments. The Appraisal Journal, v.46, n.4, p.529-537. Oct. 1978. 
JORION, P. Value at risk: a nova fonte de referência para a gestão do risco financeiro. 2.ed. São Paulo: Bolsa de Mercadorias \& Futuros, 2003. $487 \mathrm{p}$.

KLUTHCOUSKI, J.; STONE, L. F.; AIDAR, H. (Ed.). Integração lavoura-pecuária. Santo Antônio de Goiás: Embrapa Arroz e Feijão, 2003.570p.

KONDAPANENI, R. A study of the delta normal method of measuring VaR. 2005. 87f. Thesis (Master in Financial Mathematics) Worcester Polytechnic Institute, Worcester, 2005.

MANFREDO, M. R.; LEUTHOLD, R. M. Agricultural applications of Value-at-Risk analysis: a perspective. Champaign: University of Illinois at Urbana, 1998. 16p. (Office for Futures and Options Research, n.98-04).

MANFREDO, M. R.; LEUTHOLD, R. M. Market risk measurement and the cattle feeding margin: an application of Value-at-Risk. Champaign: University of Illinois at Urbana, 1999.30p. (Office for Futures and Options Research, n.99-04).

MARKOWITZ, H. M. Portfolio selection. The Journal of Finance, v.7, n.1, p.77-91. Mar. 1952.

MORAES, A. de; CARVALHO, P. C. de F.; PELISSARI, A.; ALVES, S. J.; LANG, C. R. Sistemas de integração lavoura-pecuária no subtrópico da América do Sul: exemplos do Sul do Brasil. In.: SIMPÓSIO INTERNACIONAL EM INTEGRAÇÃO LAVOURA-PECUÁRIA, 2007. Palestras..., Curitiba: UFPR/UFRGS/OHIO STATE, 2007. 27p. 1 CD-ROM.

MOURA, A. D. de. Avaliação de projetos sob condições de risco utilizando o @ RISK. Viçosa: DER/UFV, 2004. 16p. (Apostila Didática). 
POPP, M.; RUDSTROM, M. Crop enterprise diversification and speciality crops. Agricultural Finance Review, v.60, n.1, p.85-98. 2000.

\title{
REILLY, F. K.; BROWN, K. C. Investment analysis and portfolio
} management. 7.ed. Cincinnati: Thomson-South Western. 2003. 1162p.

SANTOS, H. P. dos; REIS, E. M.; DERPSCH, R. Rotação de culturas. In: Embrapa Trigo, FUNDACEP-FECOTRIGO, FUNDAÇÃO ABC. Plantio direto no Brasil. Passo Fundo: Aldeia Norte, 1993. p.85-103.

TRAVERS, F. J. Investment manager analysis: a comprehensive guide to portfolio selection, monitoring, and optimization. New Jersey: John Wiley \& Sons, 2004. 384p.

\begin{abstract}
In the Brazilian agriculture, there is a great diversity and heterogeneity of production systems that are subject to wide number of factors of risks. Although these risks cannot be completely eliminated, they can be minimized by adopting some strategies, as the diversification with agricultural and cattle activities. Based on the research historical results of the Paraná's agriculture e with the measurement of the value-at-risk, this work aimed to analyze the volatility of the economic returns of integrated crop-beef cattle systems compared to systems characterized by grain cultivation or beef cattle specialization. As results, we observed that, in the short-term, the integrated crop-livestock system is the alternative that trends to generate better economic results.
\end{abstract}

Keywords: farm systems, risk, value-at-risk. 
REVISTA DE ECONOMIA E AGRONEGÓCIO, VOL.7, $N^{\circ} 2$ 